\title{
REFLECTION SYMMETRIC ERDÉLYI-KOBER TYPE OPERATORS - A QUASI-PARTICLE INTERPRETATION
}

\author{
Richard Herrmann
}

\begin{abstract}
Reflection symmetric Erdélyi-Kober type fractional integral operators are used to construct fractional quasi-particle generators. The eigenfunctions and eigenvalues of these operators are given analytically. A set of fractional creation- and annihilation-operators is defined and the properties of the corresponding free Hamiltonian are investigated. Analogue to the classical approach for interacting multi-particle systems the results are interpreted as a fractional quantum model for a description of residual interactions of pairing type.

Key Words and Phrases: Generalized fractional calculus, shifted Riesz integrals, Erdélyi-Kober integrals, fractional operators, Fock space, quasiparticle, pairing-Hamiltonian.
\end{abstract}

\section{Introduction}

The physical interpretation of operators used within the framework of fractional calculus is a vividly discussed area of actual research [15, 21, 13. One complication is the fact, that different integral- and differential operators are equally reasonable and different levels of complexity are involved, like e.g. Riesz- [22, 10] and Erdélyi-Kober [9, 17, 23, 20] integrals respectively. 
The Riesz integral ${ }_{\mathrm{Rz}} I^{\alpha}$ is given as the convolution integral, depending on a single fractional parameter $\alpha$ :

$$
\begin{aligned}
\mathrm{RZ}^{\alpha} f(x) & =\frac{1}{2 \sin (\pi \alpha / 2) \Gamma(1-\alpha)} \int_{-\infty}^{\infty} d \xi d^{-\alpha} f(\xi) \\
& =\frac{1}{2 \sin (\pi \alpha / 2) \Gamma(1-\alpha)} \int_{-\infty}^{\infty} d \xi|x-\xi|^{-\alpha} f(\xi), 0<\alpha<1
\end{aligned}
$$

with a weakly singular kernel of power-law type, where $d=|x-\xi|$ is a measure of distance on $R^{1}$.

In the following we will discuss a specific generalization of this integral, based on a Cassini-type kernel, which is the product of two measures of distance and is considered as a symmetric extension of the one-dimensional Erdélyi-Kober type integral. A family of generalized fractional integrals of this type has been introduced recently [13].

In this paper, we will derive the eigenvalues and eigenfunctions of this operator. We will collect arguments in support of the idea, that there is a close relationship between the proposed generalized fractional operator and the concept of quasi-particle operators, widely used in solid states physics, in the theory of super-conductivity and in nuclear physics respectively to model pairing effects.

For that purpose, we will first introduce the symmetric one-dimensional Erdélyi-Kober type integral and give an analytic derivation of its main properties. We will present reasonable definitions of corresponding creationand annihilation-operators and finally investigate the properties of the free Hamiltonian based on these generalized fractional operators and interpret its properties in close analogy to Cooper-pairs in momentum representation.

\section{Eigenfunctions and eigenvalues of the reflection symmetric one-dimensional Erdélyi-Kober type integral}

We will investigate a special case of the one-dimensional Erdélyi-Kober type integral, which contains two foci of the form

$$
{ }_{\mathrm{EK}} I^{\alpha, \gamma} f(x)={ }_{\mathrm{EK}} \mathcal{N} \int_{-\infty}^{\infty} d \xi d_{1}^{-\alpha / 2} d_{2}^{-\gamma / 2} f(\xi), \quad 0<\alpha+\gamma<2
$$

where

$$
d_{i}=\left|x_{i}-\xi\right| \quad i=1,2
$$

determines the distance between a focal point $x_{i}$ and a position $\xi,|\cdot|$ denotes the absolute value and the normalization factor ${ }_{\mathrm{EK}} \mathcal{N}$ is chosen such

$$
{ }_{\mathrm{EK}} \mathcal{N}=\frac{1}{2 \sin (\pi(\alpha / 4+\gamma / 4)) \Gamma(1-\alpha / 2-\gamma / 2)}
$$


that in the case $\gamma \rightarrow 0$ up to a scaling in $\alpha$ the integral coincides with the Riesz integral definition.

Setting $\gamma=\alpha$ and defining the focal points $x_{i}$ by introducing their distance $p$ via the reflection symmetric relation with respect to $x$

$$
x_{1}=x-\frac{p}{2} \quad x_{2}=x+\frac{p}{2}
$$

we obtain a reflection symmetric one-dimensional Erdélyi-Kober type integral operator with Cassini kernel type:

$$
\begin{aligned}
\mathrm{EK} I^{\alpha, p} f(x)= & \frac{1}{2 \sin (\pi \alpha / 2) \Gamma(1-\alpha)} \times \\
& \int_{-\infty}^{\infty} d \xi\left(\left|x-\frac{p}{2}-\xi\right|^{-\alpha / 2}\left|x+\frac{p}{2}-\xi\right|^{-\alpha / 2}\right) f(\xi) \\
& p \in \mathbb{R}, 0<\alpha<1
\end{aligned}
$$

which in the limit $p \rightarrow 0$ reduces to the Riesz fractional integral.

The eigenfunctions of this operator are determined by the integral equation

$$
\mathrm{EK}^{\alpha, p} \psi(x)=\Theta(k, p) \psi(x)
$$

and are both given as trigonometric functions $\psi(x)=\{\cos (k x), \sin (k x)\}, k \in$ $\mathbb{R}$. The corresponding eigenvalues $\Theta(k, p)$ result with the help of (4.3.17, 9.1.20, 9.1.24) from [1] in

$$
\begin{aligned}
\Theta(k, p)=\frac{\sqrt{\pi} \Gamma(1-\alpha / 2)}{2 \sin (\pi \alpha / 2) \Gamma(1-\alpha)} \times|k / p|^{(\alpha-1) / 2} \times \\
\left(J_{(1-\alpha) / 2}(|k p| / 2)-Y_{(\alpha-1) / 2}(|k p| / 2)\right) \\
k, p \in \mathbb{R}, 0<\alpha<1
\end{aligned}
$$

where $J_{\beta}(z)$ and $Y_{\beta}(z)$ denote the corresponding standard Bessel functions. The following asymptotic cases clarify the functional behavior of the eigenvalue spectrum $\Theta(k, p)$ :

- Limit for $p \rightarrow 0$

A series expansion of the eigenvalue spectrum for small $p$ yields:

$$
\begin{aligned}
\Theta(k, p) \approx & |k|^{\alpha-1}-|p|^{1-\alpha} \frac{4^{\alpha} \pi \sin ^{2}(\pi \alpha / 4)}{\sin (\pi \alpha) \Gamma((1-\alpha) / 2) \Gamma((3-\alpha) / 2)} \\
& +o\left(|p|^{2-\alpha}\right) \quad k, p \in \mathbb{R},|p| \ll 1,0<\alpha<1
\end{aligned}
$$

which is well behaved for small $p$ within the range of allowed $\alpha$ values and therefore a smooth transition to the eigenvalue spectrum of the Riesz integral results for vanishing $p$ :

$$
\lim _{p \rightarrow 0} \Theta(k, p)=|k|^{\alpha-1} \quad k, p \in \mathbb{R}, 0<\alpha<1
$$




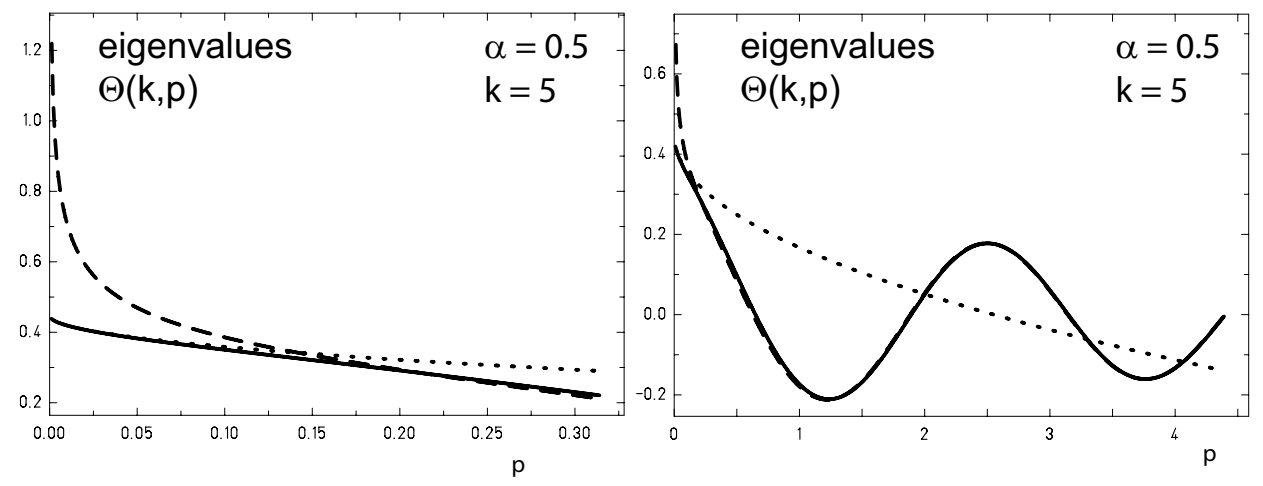

FiguRE 1. Eigenvalue as a function of the distance $p$. On the left for small $p$, on the right for larger $p$. Thick line indicates the exact solution (9), pointed line the low-distance approximation (10) and dashed line the asymptotic expansion (12).

- Limit for $|k p| \rightarrow \infty$

The asymptotic expansion of the eigenvalue spectrum for $|k p| \gg 1$, which includes the cases $k \gg 1$ and $p$ fixed and $p \gg 1$ and $k$ fixed, results according to $(9.2 .1,9.2 .2)$ from [1] in

$$
\begin{aligned}
\Theta^{\infty}(k, p) \approx & \frac{\Gamma(1-\alpha / 2)}{\sin (\pi \alpha / 4) \Gamma(1-\alpha)}|k|^{\alpha / 2-1}|p|^{-\alpha / 2} \cos (k p / 2) \\
& +o\left(|k p|^{-1}\right) \quad k, p \in \mathbb{R},|k p| \gg 1,0<\alpha<1
\end{aligned}
$$

which determines a damped oscillatory behavior for increasing $|k p|$.

- Special case $p=1 / k$

A remarkable property of the eigenvalue spectrum results from the periodicity of the trigonometric functions. For the special case $p=$ $1 / k$ the eigenvalue spectrum is given by

$$
\begin{aligned}
\Theta(k, 1 / k)= & \frac{\sqrt{\pi} \Gamma(1-\alpha / 2)}{2 \sin (\pi \alpha / 2) \Gamma(1-\alpha)} \times|k|^{(\alpha-1)} \times \\
& \quad\left(J_{(1-\alpha) / 2}(1 / 2)-Y_{(\alpha-1) / 2}(1 / 2)\right) \\
= & c(\alpha)|k|^{(\alpha-1)} \quad k \in \mathbb{R}, 0<\alpha<1
\end{aligned}
$$

and therefore coincides up to a factor $c(\alpha)$ with the Riesz fractional integral eigenvalue spectrum determined for trigonometric functions. Consequently the use of different kernel functions may lead to similar spectra. 
In figure 1 we have plotted the eigenvalue spectrum and the corresponding approximations. In case $k p>1$ the asymptotic expansion (12) is a good approximation to the exact solution, which shows an oscillatory behavior as a function of $k$ and $p$ respectively.

For a direct explanation, we consider this behavior dominated by the result of an interference of two contributions, since with

$$
\cos \left(k\left(x-\frac{p}{2}\right)\right)+\cos \left(k\left(x+\frac{p}{2}\right)\right)=2 \cos (k p / 2) \cos (k x)
$$

the asymptotic limit (12) may be rewritten as

$$
\begin{aligned}
& \Theta^{\infty}(k, p) \cos (k x)= \\
& \frac{1}{2}\left(\frac{\Gamma(1-\alpha / 2)}{\sin (\pi \alpha / 4) \Gamma(1-\alpha)}|k|^{\alpha / 2-1}|p|^{-\alpha / 2} \cos \left(k\left(x-\frac{p}{2}\right)\right)\right. \\
&\left.+\frac{\Gamma(1-\alpha / 2)}{\sin (\pi \alpha / 4) \Gamma(1-\alpha)}|k|^{\alpha / 2-1}|p|^{-\alpha / 2} \cos \left(k\left(x+\frac{p}{2}\right)\right)\right) \\
&+o\left(|k p|^{-1}\right) \quad k, p \in \mathbb{R},|k p| \gg 1,0<\alpha<1
\end{aligned}
$$

which may be interpreted as the result of a superposition of two shifted single focus kernel integrals of Riesz type.

In close analogy to Debye's saddle-point approximation for standard integrals [7] this can be derived in full generality from the symmetric ErdélyiKober kernel via a Taylor series expansion of $d_{1}$ at $x_{2}$ and and of $d_{2}$ at $x_{1}$ :

$$
\begin{gathered}
\left.d_{1}^{-\alpha / 2}\right|_{x_{2}}=\left.\left|x_{1}-\xi\right|^{-\alpha / 2}\right|_{x_{2}} \approx|p|^{-\alpha / 2}-o\left(|p|^{-2-\alpha / 2}\left(\xi-x_{2}\right)\right) \\
\left.d_{2}^{-\alpha / 2}\right|_{x_{1}}=\left.\left|x_{2}-\xi\right|^{-\alpha / 2}\right|_{x_{1}} \approx|p|^{-\alpha / 2}+o\left(|p|^{-2-\alpha / 2}\left(\xi-x_{1}\right)\right) \\
p \in \mathbb{R},|p| \gg 1,0<\alpha<1
\end{gathered}
$$

and therefore

$$
\begin{aligned}
\mathrm{EK}^{\alpha, p} f(x)= & { }_{\mathrm{EK}} \mathcal{N} \int_{-\infty}^{\infty} d \xi d_{1}^{-\alpha / 2} d_{2}^{-\alpha / 2} f(\xi) \\
\approx & \mathrm{EK} \mathcal{N} \int_{-\infty}^{\infty} d \xi\left(d_{1}^{-\alpha / 2}|p|^{-\alpha / 2}+d_{2}^{-\alpha / 2}|p|^{-\alpha / 2}\right) f(\xi) \\
= & { }_{\mathrm{EK}} \mathcal{N}|p|^{-\alpha / 2}\left(\int_{-\infty}^{\infty} d \xi d_{1}^{-\alpha / 2} f(\xi)+\int_{-\infty}^{\infty} d \xi d_{2}^{-\alpha / 2} f(\xi)\right) \\
= & |p|^{-\alpha / 2}{ }_{\mathrm{EK}} \mathcal{N} \int_{-\infty}^{\infty} d \xi|x-\xi|^{-\alpha / 2} f(\xi+p / 2) \\
& +|p|^{-\alpha / 2}{ }_{\mathrm{EK}} \mathcal{N} \int_{-\infty}^{\infty} d \xi|x-\xi|^{-\alpha / 2} f(\xi-p / 2) \\
& \quad p \in \mathbb{R},|p| \gg 1,0<\alpha<1
\end{aligned}
$$

which is up to a factor the sum of two shifted Riesz integrals. 
This observation directly leads to a physical interpretation of the symmetric Erdélyi-Kober integral operator in terms of a two-particle operator.

\section{Creation- and annihilation-operators of symmetric Erdélyi-Kober type}

Let us define the non-local pendant ${ }_{\mathrm{C}} \hat{O}$ of Caputo type of a local spacedependent operator loc $\hat{o}$ based on the symmetric Erdélyi-Kober type integral (3) as

$$
{ }_{\mathrm{C}} \hat{O}={ }_{\mathrm{EK}} I^{\alpha, \gamma}{ }_{\mathrm{loc}} \hat{O}
$$

and of Riemann type with inverted operator sequence

$$
{ }_{\mathrm{R}} \hat{O}={ }_{\operatorname{loc}} \hat{o}_{\mathrm{EK}} I^{\alpha, \gamma}
$$

The quantization of a multi-particle system may be realized on a Fockspace introducing creation- $\left(\hat{c}^{\dagger}\right)$ and annihilation- $(\hat{c})$ operators acting on a vacuum state $\mid 0>$, which is characterized by the condition:

$$
\hat{c} \mid 0>=0
$$

In occupation number representation the Fock-space is constructed by repeated action of the creation-operator on a given state $|n\rangle$

$$
\begin{aligned}
\hat{c}^{\dagger} \mid n> & =\sqrt{n+1} \mid n+1> \\
\hat{c} \mid n> & =\sqrt{n} \mid n-1>
\end{aligned}
$$

We may therefore introduce the following fractional creation and annihilationoperators:

According to (23) we define a set of Caputo type operators in space representation

$$
\begin{aligned}
{ }_{\mathrm{C}} \hat{C}^{\dagger} & ={ }_{\mathrm{EK}} I^{\alpha, \gamma} \hat{c}^{\dagger} \\
{ }_{\mathrm{C}} \hat{C} & ={ }_{\mathrm{EK}} I^{\alpha, \gamma} \hat{c}
\end{aligned}
$$

and we introduce a set of Riemann type operators in space representation according to (24)

$$
\begin{aligned}
{ }_{\mathrm{R}} \hat{C}^{\dagger} & =\hat{c}^{\dagger}{ }_{\mathrm{EK}} I^{\alpha, \gamma} \\
{ }_{\mathrm{R}} \hat{C} & =\hat{c}_{\mathrm{EK}} I^{\alpha, \gamma}
\end{aligned}
$$

The corresponding non-local vacuum state is determined by the condition:

$$
\begin{aligned}
& { }_{\mathrm{C}} \hat{C} \mid 0>{ }_{\mathrm{C}}=0 \\
& { }_{\mathrm{R}} \hat{C} \mid 0>_{\mathrm{R}}=0
\end{aligned}
$$

where $\mid 0>_{\mathrm{C}}$ and $\mid 0>_{\mathrm{R}}$ are the vacuum states associated with the Caputo- and Riemann-type operators, respectively. 


\section{SYMMETRIC ERDÉLYI-KOBER TYPE OPERATORS}

It should be noted, that for the Caputo type set of operators (28) the vacuum state $\mid 0>_{\mathrm{C}}$ is identical with its local pendant. For the Riemann type operators, in general the corresponding non-local vacuum state differs from its local pendant.

\section{Quasi-particle interpretation}

In literature, classical applications of fractional calculus have been widely studied for time-like and space-like variables $x$, see e.g. [14, 21, 12].

The transition from classical mechanics to quantum mechanics may be interpreted as a transition from independent coordinate space and momentum space first to a Hilbert and then to a Fock space respectively, where space and momentum operators are treated similarly as a canonical coordinate set.

Consequently a postulate of quantum mechanics states, that results must be independent of the specific choice of a representation e.g. space or momentum representation. This is the mathematical manifestation of wave-particle-duality: The behavior of a given quantum object may be described completely using e.g. either the position $x$ or wave vector $k$ operator respectively.

As a direct consequence, a fractional extension of standard quantum mechanics has to be formulated on a dual space. A treatment of timeand/or space dependent problems in fractional quantum mechanics using fractional operators e.g. Erdélyi-Kober type operators is therefore based on the following representation pairs:

coordinate: time $t$, conjugate coordinate: energy $E$ :

$$
\{t, E\}=\left\{t,-i \hbar \partial_{t}\right\} \rightarrow{ }_{\mathrm{EK}} I^{\alpha, \gamma}(t)\left\{t,-i \hbar \partial_{t}\right\}
$$

For time-like coordinates the focal points $x_{1} \equiv t_{1}$ and $x_{2} \equiv t_{2}$ determine system properties earlier or later with respect to present $x \equiv t$, which touches the question of causal and anti-causal event sequences. The corresponding non-local phenomena are memoryor hysteresis-effects and are observed e.g. in magnetization curves of ferro-magnets [19].

In order to obey the correct sequence of cause and effect the symmetric Erdélyi-Kober type operator may be used to describe particle / anti-particle pairs [12].

Since according to de Broglie the energy of a quantum object is directly related to a frequency via

$$
E=\hbar \omega=h \nu
$$


We may alternatively consider:

coordinate: frequency $\nu$, conjugate coordinate: time $t$ :

$$
\{\nu, t\}=\left\{\nu,-i h \partial_{\nu}\right\} \rightarrow \mathrm{EK}^{\alpha, \gamma}(\nu)\left\{\nu,-i h \partial_{\nu}\right\}
$$

In frequency representation, which is the canonically conjugate to time representation, the points $x_{1} \equiv \nu_{1}$ and $x_{2} \equiv \nu_{2}$ mark the properties of lower or higher frequency with respect to $x \equiv \nu$. Corresponding non-local concepts base on deviations of temporal coherence phenomena, which results in temporal interference or in the case of light, the deviation from monochromaticity.

A key experiment is the Michelson-Morley-experiment, which failed to demonstrate the effect on the speed of light relative to the ether. [18].

coordinate: space $x$, conjugate coordinate: momentum $k$ :

$$
\{x, k\}=\left\{x,-i \hbar \partial_{x}\right\} \rightarrow{ }_{\mathrm{EK}} I^{\alpha, \gamma}(x)\left\{x,-i \hbar \partial_{x}\right\}
$$

For space-like coordinates the points $x_{1}, x_{2}$ mark a position left or right with respect to $x$. The corresponding phenomena are nonlocal concepts also known historically as action-at-a-distance, which was suspected for centuries by physicists and seemed obsolete after Maxwell introduced fields instead of forces, see also [8].

In quantum mechanics it is known as quantum entanglement and marks the fact, that quantum numbers of quantum systems are correlated over large distances.

A key-experiment on non-locality is e.g. the Aharonov-Bohm-effect [2]. In [13 we suggested, to use the symmetric Erdélyi-Kober type operator to describe the behavior of symmetric mesons, like strangeonium, charmonium and bottomoniom respectively.

An alternative representation is:

coordinate: momentum $k$, conjugate coordinate: space $x$ :

$$
\{k, x\}=\left\{k,-i \hbar \partial_{k}\right\} \rightarrow{ }_{\mathrm{EK}} I^{\alpha, \gamma}(k)\left\{k,-i \hbar \partial_{k}\right\}
$$

In momentum representation, which is the canonically conjugate to coordinate representation, the points $x_{1} \equiv k_{1}$ and $x_{2} \equiv k_{2}$ mark properties of slower or faster with respect to actual momentum $x \equiv k$. Corresponding non-local concepts base on deviations of spatial coherence phenomena, which result in spatial interference. A key experiment is Young's interference/double slit experiment [24]. 


\section{SYMMETRIC ERDÉLYI-KOBER TYPE OPERATORS}

In case of angular momentum space, which may be considered the canonically conjugate to spherical coordinate space e.g. in two dimensions the points $x_{1} \equiv m_{1}$ and $x_{2} \equiv m_{2}$ mark properties of slower or faster rotation with respect to actual angular momentum $x \equiv m$.

In this section, we will give an example of a reasonable application of the above introduced Erdélyi-Kober type derivatives in terms of a momentum representation characterized by quantum numbers $k, p$.

We will use the derived operators for a description of quantum multiparticle systems and apply the concept of second quantization to model effects, which are observed e.g. in laser physics as laser-electron interaction (excitons) [16], in low temperature physics as superconductivity (Cooperpairs) [6, 3] and in nuclear physics as the pairing gap, which is observed as an odd-even effect in the binding energies of nucleons (pairing) [4] or the back-bending effects for high-spin states in nuclei respectively [11], only to name a few.

We will collect arguments which support the approach, that the properties of the hitherto presented complex fractional operators may be directly understood within the framework of such models.

We will investigate the properties of the diagonal Hamiltonian $H$

$$
H=\sum_{k, p>0} E_{k, p} \hat{C}_{p}^{\dagger}(k) \hat{C}_{p}(k)
$$

where the non-local creation- and annihilation-operators of symmetric ErdélyiKober type are explicitly given e.g. for Caputo type operators

$$
\begin{aligned}
\hat{C}_{p}^{\dagger}(k)= & \frac{1}{2 \sin (\pi \alpha / 2) \Gamma(1-\alpha)} \times \\
& \int_{-\infty}^{\infty} d \kappa\left(\left|k-\frac{p}{2}-\kappa\right|^{-\alpha / 2}\left|k+\frac{p}{2}-\kappa\right|^{-\alpha / 2}\right) \hat{c}_{\kappa}^{\dagger} \\
\hat{C}_{p}(k)= & \frac{1}{2 \sin (\pi \alpha / 2) \Gamma(1-\alpha)} \times \\
& \int_{-\infty}^{\infty} d \kappa\left(\left|k-\frac{p}{2}-\kappa\right|^{-\alpha / 2}\left|k+\frac{p}{2}-\kappa\right|^{-\alpha / 2}\right) \hat{c}_{\kappa} \\
& p \in \mathbb{R}, 0<\alpha<1
\end{aligned}
$$

which we will use in the following derivation. Since the procedure for Riemann-type operators according (30) leads to similar results, it will be omitted here. 
For $p \gg 1$ we may replace the integral according to (19) by

$$
\begin{aligned}
\hat{C}_{p}^{\dagger}(k) \approx & \frac{1}{2 \sin (\pi \alpha / 2) \Gamma(1-\alpha)} \times \\
& p^{-\alpha / 2} \int_{-\infty}^{\infty} d \kappa\left(\left|k-\frac{p}{2}-\kappa\right|^{-\alpha / 2}+\left|k+\frac{p}{2}-\kappa\right|^{-\alpha / 2}\right) \hat{c}_{\kappa}^{\dagger} \\
= & \frac{1}{2 \sin (\pi \alpha / 2) \Gamma(1-\alpha)} \times \\
& p^{-\alpha / 2} \int_{-\infty}^{\infty} d \kappa\left(|k-\kappa|^{-\alpha / 2} \hat{c}_{\kappa-\frac{p}{2}}^{\dagger}+|k-\kappa|^{-\alpha / 2} \hat{c}_{\kappa+\frac{p}{2}}^{\dagger}\right) \\
= & \frac{p^{-\alpha / 2}}{2 \sin (\pi \alpha / 2) \Gamma(1-\alpha)} \int_{-\infty}^{\infty} d \kappa|k-\kappa|^{-\alpha / 2}\left(\hat{c}_{\kappa-\frac{p}{2}}^{\dagger}+\hat{c}_{\kappa+\frac{p}{2}}^{\dagger}\right)
\end{aligned}
$$

and similarly

$$
\hat{C}_{p}(k) \approx \frac{p^{-\alpha / 2}}{2 \sin (\pi \alpha / 2) \Gamma(1-\alpha)} \int_{-\infty}^{\infty} d \kappa|k-\kappa|^{-\alpha / 2}\left(\hat{c}_{\kappa-\frac{p}{2}}+\hat{c}_{\kappa+\frac{p}{2}}\right)
$$

We now introduce four operators of shifted Riesz-Caputo-type in momentum space via

$$
\begin{aligned}
& \hat{A}_{p}^{\dagger}(k)=\frac{1}{2 \sin (\pi \alpha / 4) \Gamma(1-\alpha / 2)} \int_{-\infty}^{\infty} d \kappa|k-\kappa|^{-\alpha / 2} \hat{c}_{\kappa+\frac{p}{2}}^{\dagger} \\
& \hat{A}_{p}(k)=\frac{1}{2 \sin (\pi \alpha / 4) \Gamma(1-\alpha / 2)} \int_{-\infty}^{\infty} d \kappa|k-\kappa|^{-\alpha / 2} \hat{c}_{\kappa+\frac{p}{2}} \\
& \hat{B}_{p}^{\dagger}(k)= \frac{1}{2 \sin (\pi \alpha / 4) \Gamma(1-\alpha / 2)} \int_{-\infty}^{\infty} d \kappa|k-\kappa|^{-\alpha / 2} \hat{c}_{\kappa-\frac{p}{2}}^{\dagger} \\
& \hat{B}_{p}(k)=\frac{1}{2 \sin (\pi \alpha / 4) \Gamma(1-\alpha / 2)} \int_{-\infty}^{\infty} d \kappa|k-\kappa|^{-\alpha / 2} \hat{c}_{\kappa-\frac{p}{2}} \\
& p \in \mathbb{R}, 0<\alpha<2
\end{aligned}
$$

where

$$
\begin{aligned}
& \hat{B}_{p}^{\dagger}(k)=\hat{A}_{-p}^{\dagger}(k) \\
& \hat{B}_{p}(k)=\hat{A}_{-p}(k)
\end{aligned}
$$

which defines a new class of shifted single particle operators and is a subject of interest for future research by its own. 
As a consequence we obtain the relation

$$
\begin{gathered}
\hat{C}_{p}^{\dagger}(k)=\frac{p^{-\alpha / 2} \sin (\pi \alpha / 4) \Gamma(1-\alpha / 2)}{\sin (\pi \alpha / 2) \Gamma(1-\alpha)}\left(\hat{A}_{p}^{\dagger}(k)+\hat{B}_{p}^{\dagger}(k)+R(k, p)\right) \\
\hat{C}_{p}(k)=\frac{p^{-\alpha / 2} \sin (\pi \alpha / 4) \Gamma(1-\alpha / 2)}{\sin (\pi \alpha / 2) \Gamma(1-\alpha)}\left(\hat{A}_{p}(k)+\hat{B}_{p}(k)+R(k, p)\right) \\
p \in \mathbb{R}, 0<\alpha<1
\end{gathered}
$$

with a residual interaction term $R(k, p)$, which vanishes for $p \rightarrow \infty$.

With these definitions, we obtain for the Hamiltonian (39) :

$$
\begin{aligned}
H \approx & \frac{\sin ^{2}(\pi \alpha / 4) \Gamma^{2}(1-\alpha / 2)}{\sin ^{2}(\pi \alpha / 2) \Gamma^{2}(1-\alpha)} \times \\
& \sum_{k, p>0} E_{k, p} p^{-\alpha}\left(\hat{A}_{p}^{\dagger} \hat{A}_{p}+\hat{B}_{p}^{\dagger} \hat{B}_{p}+G_{k, p}\left(\hat{A}_{p}^{\dagger} \hat{B}_{p}+\hat{B}_{p}^{\dagger} \hat{A}_{p}\right)\right)
\end{aligned}
$$

where the constant factor $G_{k, p} \neq 1$ emulates the influence of the residual interaction $R(k, p)$.

This is a special case of the general pairing Hamiltonian [3, [4, [16]

$$
H_{\text {pair }}=\sum_{k} E_{k} \hat{a}_{k}^{\dagger} \hat{a}_{k}+\omega_{k} \hat{b}_{k}^{\dagger} \hat{b}_{k}+g_{k}\left(\hat{a}_{k}^{\dagger} \hat{b}_{k}+\hat{b}_{k}^{\dagger} \hat{a}_{k}\right)
$$

which by performing a Bogoliubov transformation [5] may be exactly diagonalized into [1]

$$
H_{\text {pair }}^{\prime}=\sum_{k>0} \Omega_{k}\left(\hat{\beta}_{k}^{\dagger} \hat{\beta}_{k}+\hat{\beta}_{-k}^{\dagger} \hat{\beta}_{-k}\right)
$$

where the annihilation-operators $\hat{\beta}_{k}$ are given as

$$
\begin{aligned}
\hat{\beta}_{k} & =u_{k} \hat{a}_{k}+v_{k} \hat{b}_{k}^{\dagger} \\
\hat{\beta}_{-k} & =u_{k} \hat{b}_{k}+v_{k} \hat{a}_{k}^{\dagger}
\end{aligned}
$$

and the corresponding creation operators $\hat{\beta}_{k}^{\dagger}$ :

$$
\begin{aligned}
\hat{\beta}_{k}^{\dagger} & =u_{k} \hat{a}_{k}^{\dagger}-v_{k} \hat{b}_{k} \\
\hat{\beta}_{-k}^{\dagger} & =u_{k} \hat{b}_{k}^{\dagger}+v_{k} \hat{a}_{k}
\end{aligned}
$$

where $v_{k}^{2}, u_{k}^{2}$ with $u^{2}+v^{2}=1$ are the occupation probabilities for a particle/hole state and $\Omega_{k}$ is the quasi-particle energy of a particle-hole state.

We may therefore interpret objects, which are generated using symmetric Erdélyi-Kober type creation and annihilation-operators as compounds of Riesz type objects, which we call quasi-particles in analogy to the classical case of a pairing Hamiltonian. 
A fractional Cooper-pair of this type may then be characterized by a coherent momentum vector $k$, where its two components of Riesz type are determined by the two momenta $k \pm p / 2$ coupled to $k$.

In nuclear physics the difference in binding energies for even and odd nuclei is explained due to a residual interaction of pairing type for protons and neutrons respectively [4]. In this case it is tempting to associate $k$ with the Fermi-level $k_{F}$, where $p$ determines the momentum of a given particle-hole pair.

Using symmetric Erdélyi-Kober type integrals, a fundamental new aspect in treating pairing effects emerges within the concept of fractional calculus. The pairing effects are modeled via a Cassini- type kernel function, which allows a new analytical approach.

\section{Conclusion}

We have derived the eigenfunctions and eigenvalues of a reflection symmetric Erdélyi-Kober type integral. In terms of a non-local quasi-particle Hamiltonian we have investigated the properties of this operator and found strong analogies to the quasi-particle concept used to describe pairing effects in e.g. solid states and nuclear physics.

Symmetric Erdélyi-Kober type integrals allow a direct physical interpretation in terms of a quasi-particle operators, where two momenta are coupled to $p=0$.

Even more important, within the framework of fractional calculus, these operators allow a new approach for an alternative treatment of multiparticle quantum systems, where general pairing effects are modeled analytically using a Cassini-type kernel function.

This opens a new promising area of future research within the framework of generalized fractional calculus.

\section{Acknowledgements}

We thank A. Friedrich and V. S. Kiryakova for useful discussions and suggestions.

\section{References}

[1] M. Abramowitz and I. A. Stegun, Handbook of Mathematical Functions Dover Publications, New York (1965).

[2] Y. Aharonov and D. Bohm, Significance of electromagnetic potentials in the quantum theory Phys. Rev. Lett. 115 (1959) 485-491.

[3] J. Bardeen, L. N. Cooper, and J. R. Schrieffer, Theory of superconductivity Phys. Rev. 108 (1957) 1175-1204. 
[4] S. T. Belyaev, Effect to pairing correlations on nuclear properties Mat. Fys. Medd. Dan. Vid. Selsk. 31 (1959).

[5] N. N. Bogoliubov and S. V. Tjablikov, Soviet J. Eks. Teor. Fiz. 19 (1949) 256.

[6] L. N. Cooper, Bound electron pairs in a degenerate Fermi gas Phys. Rev 104 (1956) 1189-1190.

[7] P. Debye, Näherungsformeln für die Zylinderfunktionen für große Werte des Arguments und unbeschränkt veränderliche Werte des Index. Mathematische Annalen 67 (1909) 535-558, English translation in P. J. W. Debye, The collected papers of Peter J. W. Debye, Interscience Publishers, Inc., New York (1954).

[8] A. Einstein, B. Podolsky and N. Rosen Can quantum-mechanical description of physical reality be considered complete? Phys. Rev. 47 (1935) 777-780.

[9] A. Erdélyi, On fractional integration and its application to the theory of Hankel transforms. The Quarterly J. of Mathematics (Oxford), Second Ser., 11 (1940), 293-303.

[10] W. Feller, On a generalization of Marcel Riesz' potentials and the semigroups generated by them. Comm. Sem. Mathem. Universite de Lund (1952), 72-81.

[11] W. Greiner and J. A. Maruhn, Nuclear Models. Springer, Heidelberg, Berlin, New York (1996).

[12] R. Herrmann, Fractional Calculus - An Introduction for Physicists, 2nd ed., World Scientific Publ., Singapore (2014).

[13] R. Herrmann, Towards a geometric interpretation of generalized fractional integrals - Erdélyi-Kober type integrals on $R^{N}$, as an example. Fract. Calc. Appl. Anal., Vol. 17, No 2 (2014), 361-370, DOI: 10.2478/s13540-014-0174-4 http://link.springer.com/article/10.2478/s13540-014-0174-4.

[14] R. Hilfer, Applications of fractional calculus in physics World Scientific Publ., Singapore (2000).

[15] V. S. Kiryakova, Generalized Fractional Calculus and Applications. Longman (Pitman Res. Notes in Math. Ser. 301), Harlow; Co-publ.: John Wiley and Sons, New York (1994).

[16] C. Kittel, Quantum Theory of Solids. Wiley (1987).

[17] H. Kober, On fractional integrals and derivatives. Quarterly J. of Mathematics (Oxford Ser.) 11, No 1 (1940), 193-211.

[18] A. A. Michelson and E. W. Morley, On the relative motion of the earth and the luminiferous ether American Journal of Science 34 (1887) 333345 . 
[19] A. Mielke and T. Roubicek, A rate-independent model for inelastic behavior of shape-memory alloys. Multiscale Model. Simul. 1 (2003) 571-597.

[20] G. Pagnini, Erdelyi-Kober fractional diffusion. Fract. Calc. Appl. Anal. 15, No 1 (2012), 117-127; DOI: 10.2478/s13540-012-0008-1; http://link.springer.com/article/10.2478/s13540-012-0008-1.

[21] I. Podlubny, Geometric and physical interpretation of fractional integration and fractional differentiation. Fract. Calc. Appl. Anal. 5, No 4 (2002), 367-386; http://www.math.bas.bg/ fcaa/; and Corrections to Figure 4 in: Fract. Calc. Appl. Anal. 6, No 1 (2003), 109-110.

[22] M. Riesz, L'integrale de Riemann-Liouville et le probléme de Cauchy. Acta Math. 81 (1949), 1-223.

[23] I.N. Sneddon, Mixed Boundary Value Problems in Potential Theory. North-Holland Publ. Co., Amsterdam (1966).

[24] T. Young, The Bakerian Lecture: On the Theory of Light and Colours. Philosophical Transactions of the Royal Society of London 92 (1802) $12-48$.

GigaHedron

Berliner Ring 80, D-63303 Dreieich, GERMANY

e-mail: herrmann@gigahedron.com

Received: July 17, 2014 